\title{
Abscisic acid enhances the immune response in Apis mellifera and contributes to the colony fitness
}

\author{
Pedro Negri ${ }^{1,2}$, Matias D. Maggi ${ }^{1}$, Leonor RamireZ ${ }^{2}$, Leonardo De Feudis ${ }^{1}$, \\ Nicolás SzWARski ${ }^{1}$, Silvina Quintana ${ }^{3}$, Marin J. Eguaras ${ }^{1}$, Lorenzo Lamattina ${ }^{2}$ \\ ${ }^{1}$ Laboratorio de Artrópodos, FCEyN, Universidad Nacional de Mar del Plata (UNMdP), Mar del Plata, Argentina \\ ${ }^{2}$ Instituto de Investigaciones Biológicas, CONICET-Universidad Nacional de Mar del Plata (UNMdP), Mar del Plata, \\ Argentina \\ ${ }^{3}$ Laboratorio de Biología Molecular, Fares Taie Instituto de Análisis, Mar del Plata, Argentina
}

Received 20 March 2014 - Revised 11 November 2014 - Accepted 11 December 2014

\begin{abstract}
The primary food of adult honey bees (Apis mellifera) is honey prepared by bees from nectar, provided by plants in order to stimulate the bee's pollination service. Nectar consists of carbohydrates, amino acids and water, as well as other minor compounds whose proportion varies among plant species and whose biological implications in the honey bee physiology require intense research. Several environmental stressors are causing the decline of bee colonies, and thereby, we tried to connect the nutritional quality of bee's diet with the strength of the bee's immune system. The phytohormone abscisic acid (ABA) is present in nectar, honey and adult honey bees. It has been demonstrated that ABA stimulates innate immune defences in animal cells. However, the influence of ABA on A. mellifera's health and fitness is unknown. Here, we show that honey bees fed with an ABA supplement in field experiments resulted in (i) the appearance of ABA in larvae and adult bees, (ii) enhanced haemocyte response to non-self recognition, (iii) improved wound healing and granulocyte and plasmatocyte activation and (iv) maximum adult bee population after the winter and increased pesticide tolerance. The results indicate that the naturally occurring compound ABA has a positive influence in honey bee immunity. ABA emerges as a potent booster of immune defence in A. mellifera and may be useful in addressing the colony losses threatening apiculture and pollination service worldwide.
\end{abstract}

Apis mellifera / abscisic acid / immune aptitude

\section{INTRODUCTION}

Pollination is vital to the maintenance of both wild plant communities and agricultural productivity (Potts et al. 2010). However, pollinators like bees are threatened by habitat fragmentation, agrochemicals,

Matias D. Maggi and Leonor Ramirez contributed equally to the manuscript.

Electronic supplementary material The online version of this article (doi:10.1007/s13592-014-0345-7) contains supplementary material, which is available to authorized users.

Corresponding author: L. Lamattina, lolama@mdp.edu.ar

Manuscript Editor: Yves Le Conte pathogens, climate change and the interactions among them (Potts et al. 2010; Bryden et al. 2013).

Before the winter, the queen reduces the laying of eggs (Harbo 1985), and thus in the winter, colonies are mainly conformed by adult bees. In practice, beekeepers supplement their beehives with syrup, either to stimulate the colony before plant flowering in the spring or to help bees to confront the winter (Decourtye et al. 2010).

Honey is made from the nectar that bees take from plants (Schaefer et al. 2004). Nectar contains mainly sugars and water and other minor compounds (Brodschneider and Crailsheim 2010) whose implications in honey bee physiology and health are not entirely understood. For instance, it has been recently reported that $p$-coumaric acid, a 
natural compound present in pollen, induces the expression of several genes related with detoxification and immune responses in Apis mellifera and functions as an enhancer of bee tolerance to pesticides (Mao et al. 2013).

Abscisic acid (ABA) is a phytohormone that regulates fundamental physiological functions in plants (Adie et al. 2007) and whose presence has been unambiguously demonstrated in nectar and honey (Lipp 1990; Tuberoso et al. 2010; Ferreres et al. 1996) as well as in adult honey bees (Lipp 1990). Even though it is known that honey bees are able to interconvert the isomers of ABA (Lipp 1990), the influence of ABA on A. mellifera physiology remains to be determined. In the last decade, evidence has been accumulated demonstrating that ABA operates in animal cells by stimulating innate immune defences such as cell migration and phagocytosis and by inducing nitric oxide (NO) and reactive oxygen species (ROS) production (Bruzzone et al. 2007, 2012). Nevertheless, there is no information regarding ABA effects on the insect immune system.

In honey bees, the physiological function and the capacity to mount a successful immunological response partly depend on the nutritional status of the organism (Brodschneider and Crailsheim 2010; Alaux et al. 2010). Honey bee immune defence relies on innate reactions that are based on a constitutively active cellular and inducible humoral immune response (Gätschenberger et al. 2013). Considerable data are available on humoral immune reactions characterized by the action of effectors' molecules such as antimicrobial peptides, complement-like proteins and the enzymatic cascades that regulate melanin formation and clotting (Strand 2008). In contrast, much less is known about the cellular immune system in honey bees comprising wound healing, phagocytosis and nodulation and encapsulation of the intruder (Gätschenberger et al. 2013). Elimination of microorganisms that enter into the insect haemocoele requires that the haemocytes recognize the invading species (Nardi et al. 2006). Once this occurs, the haemocytes transform from resting cells into activated cells that adhere and spread over the foreign surface (Nardi et al. 2006; Gillespie and Kanost 2006).
In this work, we tested the prediction that the ABA ingested by honey bees enhances their immune defence and results in a general benefit for the colony health. Our results indicate that ABA, a ubiquitous component of honey bee food, positively influences the immune response of individuals and the colony, favouring the adaptation of the population to an unfavourable environment.

\section{MATERIALS AND METHODS}

\subsection{Insects}

Field experiments were located in the Santa Paula experimental apiary on the route $226, \mathrm{~km} \mathrm{10,} \mathrm{Mar} \mathrm{del}$ Plata, Buenos Aires, Argentina. A. mellifera (A. mellifera ligustica-A. mellifera mellifera) colonies were reared in minihives as experimental units (Figure 1) as described by Keasar et al. (2007). The minihives were made of wood. The frames were $18-\mathrm{cm}$ width $\times 10$-cm height. The hives were $23 \mathrm{~cm}$ long $\times 14$ $\mathrm{cm}$ width $\times 22-\mathrm{cm}$ depth. The hives consisted of four combs and a Doolittle feeder adapted to the minihive size. Each minihive contained around 2000 bees. The minihives were designed by Lic. Leonardo De Feudis, an experienced beekeeper and queen breeder. New queens were used for each colony in field experiments in 2012 and 2013. The minihives were pre-selected and then selected to get standardized colonies according to population, storage (honey and pollen) and aggressive behaviour (Figure 1). The experiment started after 45 days of the standardization of the colonies. After the standardization process, the experiment started by replacing an old comb with a new one in the same position for all minihives, and the first syrup supplementation was applied (Figure 1). Three colonies were supplemented with syrup alone (control group), and another three colonies were supplemented with syrup containing $10 \mu \mathrm{M}\left(2.66 \mu \mathrm{g} \mathrm{g}^{-1}\right)$ of ABA (Sigma) (ABA-supplemented minihives) in 2012, and the same was repeated in 2013. The minihives were not prepared to store honey; hence, the supplemented syrup was totally consumed by bees every time that it was applied. A third group without syrup supplementation was not considered because this would severely affect the health of the colony and compromise the survival of colonies during the winter. Syrup was prepared by merging two parts of sucrose and one part of distilled water $(v / v)$. Minihives were supplemented with $300 \mathrm{~mL}$ of syrup per 
PREPARATION OF COLONIES

(I) MINI HIVE (experimental unit)

\begin{tabular}{|c|}
\hline FRAME 4 \\
\hline FRAME 3 \\
\hline FRAME 2 \\
\hline FRAME 1 \\
\hline FEEDER \\
\hline
\end{tabular}

(II) INITIAL COLONIES

PRE- SÉLECTION

FINAL SELECTION

EXPERIMENT STARTS

SELECTION CRITERIA:

POPULATION (adults and brood)

STORAGE (honey and pollen)

AGGRESSIVENESS

\section{START OF THE EXPERIMENT}

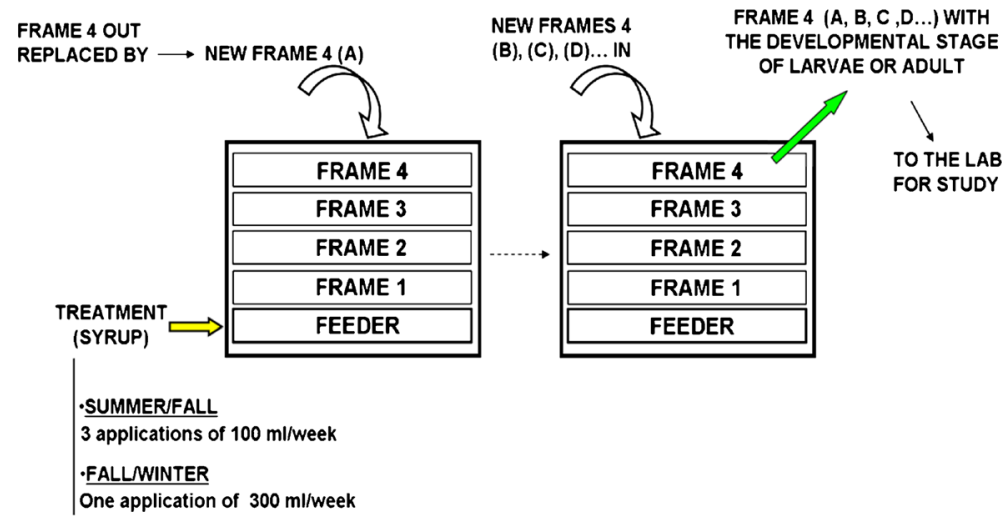

Figure 1. Experimental design for field experiments. Preparation of colonies involves minihives as experimental units $(I)$ and the selection and standardization of the initial colonies $(I I)$. Experiments consisted with the replacement of frame 4 with brood taken to the laboratory for their analysis (green arrow), by new frame 4 (white curved arrows ). Syrup supplementation is indicated by yellow arrow.

week (Figure 1), as follows: (i) the summer and the beginning of fall, three applications of $100 \mathrm{~mL}$ each, and (ii) end of fall and winter, just one application of $300 \mathrm{~mL}$ of syrup to reduce the manipulation of the minihives in the cold season. New combs containing sealed brood about to emerge or combs that contained the fifth instar (L5) larvae were removed from the colony and taken to an incubator in the lab at 30 $32{ }^{\circ} \mathrm{C}$ and $70 \% \mathrm{RH}$. Two developmental bee stages were used in the experiments: L5 larvae and newly emerged workers (Ws) that emerged in the incubator. The frames taken to the laboratory were replaced with new ones in each colony (Figure 1) in the field. These new combs included comb foundation. This procedure continued until the winter when minihives were opened once a week only for syrup supplementation. The number of combs covered with adult bees (grade) was counted in each colony to quantify the adult population before and after the winter. The maximum grade that a minihive can get is 4 and the minimum is 0 .

\subsection{Analysis of the deforming wing virus levels. RNA preparation, reverse transcription and $q P C R$}

Deforming wing virus (DWV) is transmitted by the parasitic mite Varroa destructor (Anderson and Trueman 2000) and severely affects the health of honey bees (Martin et al. 2012). Thus, during the pre-selection of the colonies to be used in the experiment, DWV levels were analyzed through reverse transcription (RT)-PCR in the colonies to standardize the incidence of the virus between the control and the ABA-supplemented groups. Before starting the experiment, the load of DWV was measured in 15 minihives. The six minihives that showed no significant differences in their DWV levels were selected to perform the experiments. Three of them were assigned for the control group, and the other three were assigned to the ABA-supplemented group.

Total RNA of ten nurse bees from each minihive was isolated using TRI-reagent (Biobasic, Inc, USA), according to the manufacturer's protocol, and digested with DNAse I Amplification Grade (Invitrogen, Carlsbad, CA, USA) for $30 \mathrm{~min}$ at $37{ }^{\circ} \mathrm{C}$ to remove any contaminating genomic DNA (gDNA). The RNA was quantified using a Rotor Gene 6000 cycler using a Quant-iT ${ }^{\mathrm{TM}}$ RiboGreen ${ }^{\circledR}$ RNA Assay Kit (Invitrogen). Complementary DNA (cDNA) was synthesized using a reaction mixture containing $1 \mu \mathrm{g}$ of total RNA, random hexamers (12 $\mathrm{ng} \mathrm{LL}^{-1}$ ) and MMLV-RT (Invitrogen), following the procedures suggested by the manufacturer. Negative controls, omitting the RNA or the RT, were included. Primers were synthesized by Eurofins MWG Operon (Huntsville, AL, USA). The sequences of the primers were DWV1 5'-CTGTATGTGGTGTGCCTG GT-3'; DWV2 5'-TTCAAACAATCCGTGAATATAG 
TGT-3' (Kukielka et al. 2008); $\beta$-actin-forward 5'ATGCCAACACTGTCCTTTCTGG-3'; $\beta$-actinreverse 5'-GACCCACCAATCCATACGGA-3' (Yang and Cox-Foster 2005).

RT-quantitative PCR (qPCR) reactions for DWV level analysis were run using EvaGreen as intercalating dye (KAPA FAST, Biosystems, Woburn, USA) and $2 \mu \mathrm{L}$ of cDNA sample in a final volume of $20 \mu \mathrm{L}$. The amplification and detection of the specific products were carried out using a Rotor Gene 6000 cycler, with the following amplification conditions: $2 \mathrm{~min}$ at $95^{\circ} \mathrm{C}$, 40 cycles of $15 \mathrm{~s}$ at $95{ }^{\circ} \mathrm{C}, 15 \mathrm{~s}$ at $58{ }^{\circ} \mathrm{C}$ and $20 \mathrm{~s}$ at $72{ }^{\circ} \mathrm{C}$. After amplification, a melting curve analysis was performed, which resulted in single product-specific melting curve. In all cases, the experiments were performed in duplicate. Negative controls for cDNA synthesis and qPCR procedures were included in all cases. RT-qPCR efficiency for $\beta$-actin and DWV PCRs was determined by a linear regression model, according to the equation $\mathrm{E}=10[-1 / \mathrm{slope}]$. DWV levels in each minihive were studied as a ratio between the values of DWV Ct (Cycle threshold) and $\beta$-actin Ct (reference gene).

\subsection{Abscisic acid extraction}

A total of 120 L5 larvae and 120 newly emerged Ws were taken for lyophilization. Twenty L5 larvae and 20 Ws were taken from each of the six colonies corresponding to the groups control and ABA-supplemented. The 20 bees were pooled for lyophilization. Three replicates were performed. ABA quantification was first performed in 2012 and repeated in 2013. Ws are bees collected immediately after the emergence; hence, they are adult bees that were not fed since they were in the stage of L5 larva. The entire midguts of Ws were extracted by pulling out from the sting of each W. The midguts were cut out and separated from the rest of the body. The rest of the $\mathrm{W}$ body was lyophilized for $\mathrm{ABA}$ quantification. This procedure was made according to Porrini et al. (2011) to avoid measuring the ABA content that could be present in the midgut due to food residues. Abscisic acid was extracted from lyophilized material using a modification of the protocol of Durgbanshi et al. (2005). Lyophilized materials, larvae and adult bees without midguts were homogenized in liquid nitrogen with a mortar and a pestle and dissolved in $5 \mathrm{~mL}$ ultra-pure water. One hundred nanograms of $\left[{ }^{2} \mathrm{H}_{6}\right]$ ABA (OlChemIm Ltd, Olomouc, Czech Republic) was added as internal standard. Extracts were transferred to $50-\mathrm{mL}$ tubes, centrifuged at $1500 \mathrm{~g}$ for $15 \mathrm{~min}$. The supernatant was collected, adjusted to $\mathrm{pH} 2.8$ with $15 \%$ $(v / v)$ acetic acid and extracted twice with an equal volume of diethyl ether.

The aqueous phase was discarded, and the organic fraction was evaporated by vacuum. Dried extracts were dissolved in $1 \mathrm{~mL}$ methanol. Samples were filtered through a syringe filter tip on a vacuum manifold at a flow rate less than $1 \mathrm{~mL} \mathrm{~min}{ }^{-1}$, and the eluate was evaporated at $358{ }^{\circ} \mathrm{C}$ under vacuum in a SpeedVac SC110 (Savant Instruments, Inc, NY, USA).

\subsection{ABA quantification by liquid chromatography-electrospray ionization and EM mass spectrometry (LC-ESI-MS-MS)}

Mass spectrometry analysis was performed on a quadruple tandem mass spectrometer (MS-MS, Quattro Ultima; Micromass, Manchester, UK) outfitted with an electrospray ion source (ESI). A mixture containing all unlabelled compounds and internal standards was separated by reversed-phase high-performance liquid chromatography (HPLC) and analyzed by tandem mass spectrometry with single ion recording (SIR) to determine retention times for all compounds. The spectrometer software MassLynxe v. 4.1 (Micromass) was used. The response was calculated as product ion peak area $\times$ (IS concentration/IS product ion peak area), where IS concentration is the known amount of the internal standard added.

HPLC was used to separate ABA extracted from honey bees. An Alliance 2695 separation module (Waters, Milford, MA, USA) equipped with a $100 \times 2.1-\mathrm{mm}, 3-\mu \mathrm{m}$ RESTEK C18 column was used to maintain performance of the analytical column. Fractions were separated using a gradient of increasing methanol concentrations, constant glacial acetic acid concentration $0.2 \%(v / v)$ in water and initial flow rate $0.2 \mathrm{~mL} \mathrm{~min}{ }^{-1}$. The gradient was increased linearly from $40 \%(v / v)$ methanol-60\% $(v / v)$ water-acetic acid at 25 min to $80 \%(v / v)$ methanol-20\% (v/v) wateracetic acid. After $1 \mathrm{~min}$, initial conditions were restored, and the system was allowed to equilibrate for $7 \mathrm{~min}$. The monitoring reaction multiple mode was used for determination of ABA. This compound was monitored at $\mathrm{m} / \mathrm{z}$ transitions of 263/153 with retention time of 9.6. The collision energy used was $15 \mathrm{eV}$ (electron volts). 
The cone voltage was $35 \mathrm{~V}$ (see Supplemental Figure 4).

\subsection{Haemolymph collection and haemocyte count}

Haemolymph collection was performed as previously described (Negri et al. 2013). Briefly, insect blood was collected from L5 larvae by puncturing the soft cuticle with a sterile $30-\mathrm{G}$ needle, and the resulting haemolymph bubble was collected with a micropipette. The cuticle was surface-sterilized with $70 \%$ ethanol before puncturing. Ws were punctured in the neck at the aorta, and haemolymph was collected from the resulting bubble. Modified plastic tubes were used to immobilize the adult honey bee and expose the aorta (Supplemental Figure 1). The haemolymph collected was transferred into a microcentrifuge tube containing ice-cold sterile Excell-405 culture medium (Sigma) (1:10 ratio of haemolymph/media). Haemolymph solution was homogenized by soft pipetting into the sampling tube and then transferred to a sterile 96-well glass bottom plate (NUNC, 96-well optical bottom plates, sterile) where all the experiments were performed. For haemocyte counting, haemolymph was collected in anticoagulant buffer in a 1:10 $(v / v)$ ratio. After gently mixing with a micropipette, haemocytes were counted using a Neubauer haemocytometer under light microscope. A total of 25 larvae and $25 \mathrm{Ws}$ were analyzed per treatment.

\subsection{Spreading of haemocytes over glass surface}

Upon recognizing a glass surface as foreign, nonadherent haemocytes transform quickly to activated adherent cells that adhere to the glass (Nardi et al. 2006). After non-self recognition, the response of insect haemocytes is to spread (Gillespie and Kanost 2006). If the foreign agent is small, this spreading ability promotes the phagocytosis of the particle, whereas a larger foreign object (or many small foreign objects) would be subjected to nodulation or encapsulation due to the cooperative action of many haemocytes (Eleftherianos et al. 2009). Inhibiting haemocyte spreading is a common strategy employed by many entomopathogens. This implies that such changes in haemocyte behaviour are important components of insect defence (Eleftherianos et al. 2009). Microbial surfaces and non-microbial and abiotic surfaces (plastic and glass) are recognized as non-self by the insect innate immune system and are encapsulated by the haemocytes. Consistently, the spreading degree of insect haemocytes on a glass surface is often used as a measure of immune fitness (Davies and Preston 1985; Dean et al. 2004). We have recently characterized haemocytes of $A$. mellifera that respond to foreign surfaces in vitro (Negri et al. 2013, 2014a, b).

Combs containing L5 larvae from the two groups (control and ABA-supplemented colonies) were taken to the lab and the L5 larvae and Ws used for the analysis. Haemolymph collection and sampling into the wells were performed as described above. After $48 \mathrm{~h}$ of culture, the number of plasmatocytes that spread near the agglomeration zones was studied. The agglomeration zone corresponds to sites where haemocytes were grouped. The haemocytes surrounding the agglomeration zones included in a frame of $3.104 \mu^{2}$ were photographed and analyzed for spreading analysis using ImageJ (NIH, USA). Ten photographed frames were analyzed per larva $(n=6)$. The percentage of plasmatocytes that spread were quantified since this is the haemocyte type showing active locomotion and pseudopodia development (Negri et al. 2014b). The experiment was repeated for three times.

For the analysis of immune cell response in newly emerged Ws, the haemolymph was collected as described above. Haemocytes were cultured over a glass surface during 6 and $24 \mathrm{~h}$ (glass challenge). Thereafter, pictures were taken, and the cellular spreading was measured using ImageJ. The haemocyte types analyzed were the ones that first adhere after contact to glass (granulocytes) (Negri et al. 2013, 2014a). A. mellifera haemocytes were observed using inverted microscopy (Nikon Eclipse Ti, $\times 60$ objectives) and differential interference contrast (DIC). Three Ws per treatment were analyzed. The experiment was repeated for three times.

\subsection{Nylon implant assays}

The nylon implants are considered an immune challenge that triggers non-self recognition in insects, followed by encapsulation and wound healing (Wilson-Rich et al. 2008; Negri et al. 2014a). To trigger a wound healing/encapsulation response, nylon threads were implanted into L5 larvae (Wilson-Rich et al. 2008; Negri et al. 2014a). The implants consisted in a nylon thread (0.155-mm diameter) knotted in the middle and 
with two sharp extremes of $1 \mathrm{~mm}$ long each. One of the extremes was introduced into the larva (parallel to the larva's skin) until the knot contacted the skin of the larva resulting in $1 \mathrm{~mm}$ of the nylon thread introduced into the larva haemocoele. The other extremes functioned as a handle for manipulation. Larvae were maintained in an incubator at $32-34{ }^{\circ} \mathrm{C}$ and $70 \%$ relative humidity. Twenty-four hours later, the implants were removed, and the larvae were used to measure haemocytic behaviour. Cells that spread upon stimulation with the glass surface were considered as immune-related haemocytes. Cellular spreading of granulocyte-like and plasmatocyte-like haemocyte types was measured using ImageJ. The different types of adherent haemocytes were analyzed as a measure of the effects generated by the nylon implant on immune-related cells. Three larvae per treatment were analyzed. The experiment was repeated for three times.

\subsection{Varroa challenge and wounding assay}

Upon injury, coagulation and melanization are characteristic responses in the wound-healing process in insects (Wilson-Rich et al. 2008). These are strong responses that are difficult to evaluate in different treatments using naïve individuals. Thereby, we took advantage of the anticoagulant capacity reported for $V$. destructor saliva (Richards et al. 2011) in order to study the effect of ABA in the wound-healing response in A. mellifera L5 larvae. L5 larvae were challenged with the Varroa mite, and then, the injury was generated with a needle. Thus, the wound-healing response was impaired due to the anticoagulant effects of $V$. destructor saliva. $V$. destructor mites were obtained from infested colonies of $A$. mellifera from our apiary. Mature mites were removed from bee broods with a moistened paint brush and placed in an incubation stove at $70 \% \mathrm{RH}$ and $33-34{ }^{\circ} \mathrm{C}$ in glass Petri dishes. The mites were submitted to starvation for $3 \mathrm{~h}$. Honey bee larvae from control and ABA-supplemented colonies were gently deposited into 24-well plates (one larva per well), and one Varroa mite was deposited on each larva. Varroa mites were deposited alone in wells without any larva (one mite per well) as a control of the mite feeding. Varroa females are very susceptible to starvation and die within $24 \mathrm{~h}$ when no larvae are supplied as food. A piece of foam of $2 \mathrm{~mm}$ thickness was placed between the plate and the lid to prevent transferring mites between the wells. Twentyfour hours after challenging larvae with Varroa, the mites were tested to be over each larva, and the viability was checked through a stereomicroscope. Thereafter, the larvae were transferred to new clean plates without mites, and an artificial wounding was performed with a sterile 30-G needle. Twenty-four hours later, the wound-healing response was analyzed under a stereo microscope. The phenotype (healthy versus sick) of each larva was also analyzed. Four larvae per treatment were challenged with Varroa parasitation. The experiment was repeated for two times.

\subsection{Protein determination and measurements of enzymatic activities}

Individuals of L5 larvae and Ws were homogenized in liquid nitrogen with a mortar and a pestle and dissolved in $700 \mu \mathrm{L}$ of $50-\mathrm{mM}$ potassium phosphate buffer, $\mathrm{pH}$ 7.0. The homogenates were centrifuged at $10,000 \mathrm{~g}$ for $10 \mathrm{~min}$, and the supernatants were employed for protein quantification and catalase (CAT) and phenoloxidase (PO) activities. Bradford assay was used for protein quantification.

CAT activity was assayed in mixtures containing $10 \mathrm{mM} \mathrm{H}_{2} \mathrm{O}_{2}$ and $20 \mu \mathrm{L}$ of extract in $50-\mathrm{mM}$ potassium phosphate buffer, $\mathrm{pH}$ 7.0. The activity was determined following the consumption of $\mathrm{H}_{2} \mathrm{O}_{2}$ (extinction coefficient of $39.4 \mathrm{mM}^{-1} \mathrm{~cm}^{-1}$ ) at $240 \mathrm{~nm}$ for 2 min using a spectrophotometer (Hitachi). Four experiments were performed for L5 larvae and Ws $(n=20)$.

$\mathrm{PO}$ is one of the main enzymes responsible for the melanization process in the insect's immune system (Gillespie and Kanost 2006; Wilson-Rich et al. 2008). PO activity is a common measure to assess humoral immune aptitude in adult bees (Wilson-Rich et al. 2008). PO was assayed through the catalysis of the conversion of L-dopa (3,4-dihydroxy-L-phenylalanine) to dopachrome, measured photometrically at $490 \mathrm{~nm}$ every hour for $3 \mathrm{~h}$. Each sample contained $300 \mu \mathrm{L} \mathrm{L}-$ dopa, $300 \mu \mathrm{L}$ of extract and $2.4 \mathrm{~mL}$ of $50-\mathrm{mM}$ potassium phosphate buffer, $\mathrm{pH}$ 7.0. $\mathrm{PO}$ activity was expressed as the rate of substrate conversion after 1 or $3 \mathrm{~h}$ in each individual sample. The assay was repeated for four times $(n=20)$.

\subsection{Bee lethality tests}

To test the ABA toxicity, we followed the methodology described in Maggi et al. (2013). W bees 
were manually fed $80 \mu \mathrm{L}$ of various concentrations of ABA: $0,50,500$ and $1000 \mu \mathrm{M}$ in $33 \%(w / v)$ syrup solution. Bees were placed inside glass vials ( $3 \mathrm{~cm}$ long $\times 1-\mathrm{cm}$ diameter) for $3 \mathrm{~h}$ without a food source. Then, a micropipette tip that contained $80 \mu \mathrm{L}$ of syrup plus the different ABA concentrations was supplied to each bee. Bees consumed the $80 \mu \mathrm{L}$ in $3 \mathrm{~h}$ average. Twenty replicates were performed per treatment. Once the supplied solution was totally consumed, the bees were fed ad libitum syrup without $\mathrm{ABA}$.

Oxalic acid (OA) is used as acaricide and can cause mortality of adult bees and brood due to its ingestion (Porrini et al. 2011; Maggi et al. 2010a). OA (Ciccarelli Laboratories ${ }^{\circledR}$, Argentina) was supplied at different concentrations $(0,1,3,5$ and $7 \%$ $w / v)$ in $80-\mu \mathrm{L}$ final volumes of syrup. The doses of OA consumed by the bees were $0,1.5,4.5,7.5$ and $10 \mathrm{mg}$, respectively, for the tested concentrations. Bees were fed syrup containing $50 \mu \mathrm{M}$ ABA (ABA) or not (control) and the different concentrations of OA in a final volume of $80 \mu \mathrm{L}$. Twenty-four hours later, lethal concentration 50 (LC50) was quantified. The tolerance assays for OA were evaluated in newly emerged bees (Ws) and nurses. Twenty replicates were performed per treatment.

The bees' tolerance to carvacrol was assessed through the complete exposure method (Porrini et al. 2011). In this method, adult $\mathrm{W}$ bees are in contact with the pesticide, and thereby, they are able to touch, smell and intake carvacrol (Aliano et al. 2006). In this case, bees were taken from the field experiment coming from the groups fed syrup (control) or with ABA-supplemented syrup (ABA). The treatment was performed in Petri dishes $(140 \times 20 \mathrm{~mm})$. Carvacrol $(2.5,5,10$ and $20 \mathrm{mg} \mathrm{mL}^{-1}$ ) was diluted in ethanol, the solution was applied to the bottom of the Petri dish, and five bees were put into the dishes. Powdered sugar with water was supplied inside the dishes for feeding bees. Complete exposure was tested for each concentration of carvacrol, and ethanol was included as control. The dishes were placed in an incubator at $33{ }^{\circ} \mathrm{C}$ and $70 \% \mathrm{RH}$. Five replicates were done for each experimental unit, and bee mortality was determined $24 \mathrm{~h}$ later. LC50 values and inverse $95 \%$ fiducial confidence limits were estimated (Maggi et al. 2011; Lindberg et al. 2000). Variables were transformed with the probity function.

\subsection{Statistics}

$t$ tests were performed for data with normal distribution. In those cases where data did not follow a normal distribution, a Mann-Whitney test was applied. An ANOVA factorial $a \times b$ test was performed for PO activity assay analysis.

\section{RESULTS}

\subsection{ABA is present in L5 larvae and newly emerged worker bees}

To evaluate the effects of ABA supplementation in bees, two groups of honey bee colonies were compared: control groups that were colonies supplemented with syrup alone and the ABA groups that were colonies fed with syrup supplemented with $10 \mu \mathrm{M}$ ABA. Figure 1 shows a diagram of the experimental design. Figure $2 \mathrm{a}$ is a scheme that shows the pathway followed by the ABA from the feeder until reaching the brood. After 2 months of initiating the experiments, the ABA content was quantified in the L5 larvae and newly emerged Ws to corroborate that the supplemented ABA ended within the brood's diet (Figure 2). Figure $2 \mathrm{~b}$ shows that L5 larvae coming from minihives fed ABA-supplemented syrup contained $40 \%$ more $\mathrm{ABA}$ than that found in control L5 larvae $(3.66 \pm 0.38$ versus 2.63 $\pm 0.31 \mathrm{nmol} \mathrm{g}^{-1}$ dry weight (DW), respectively, $t$ test, $P=0.01$, whereas Ws from the ABAsupplemented group contained $70 \%$ more ABA than the control $(28.52 \pm 4.08$ versus 17.35 $\pm 0.52 \mathrm{nmol} \mathrm{g}^{-1} \mathrm{DW}$, respectively, $t$ test, $P=0.02$ ).

\subsection{ABA activates cellular and wound- healing responses in honey bees}

To assess the haemocytic response to wounding, nylon threads were implanted into A. mellifera L5 larvae from the control and the ABA-supplemented colonies. The spreading of haemocytes was evaluated $24 \mathrm{~h}$ after implanting (Figure $3 \mathrm{a}-\mathrm{e}$ ). The cellular area of granulocytes and plasmatocytes was significantly higher in ABA-supplemented larvae before implanting (Figure $3 \mathrm{a}, \mathrm{c}$ ). A significantly higher spreading was also detected in response to implanting in 


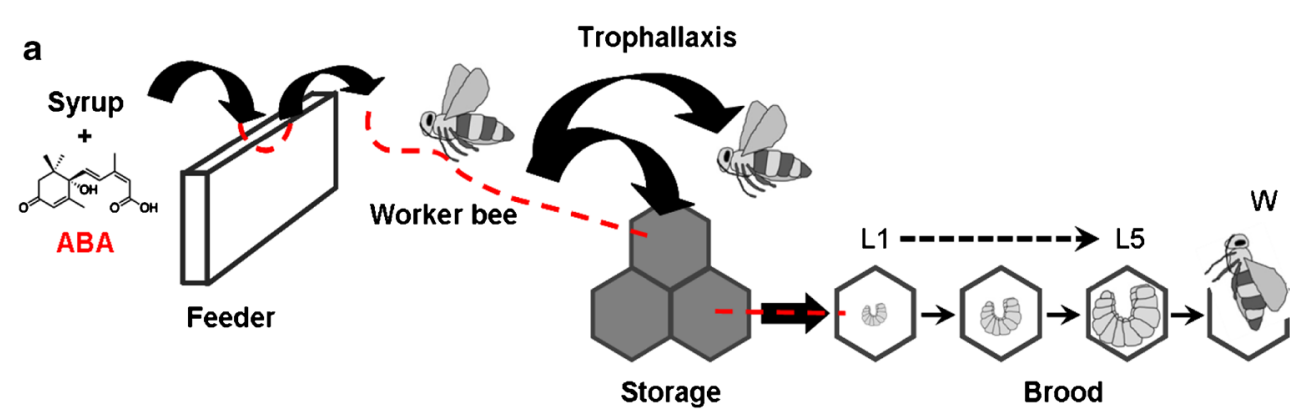

b

\begin{tabular}{ccc}
\hline & & ABA content $\left(\mathrm{nmol} \mathrm{g} \mathrm{g}^{-1} \mathrm{DW}\right)$ \\
\hline L5 Larvae & Control & $2.63 \pm 0.31(100 \%)$ \\
& ABA & $3.66 \pm 0.38(140 \%)$ \\
\hline W & Control & $17.35 \pm 0.52(100 \%)$ \\
& $A B A$ & $28.52 \pm 4.08(170 \%)$ \\
\hline
\end{tabular}

Figure 2. Supplementation of $A B A$ in minihives and $A B A$ content in larvae and adult bees. a Scheme showing the route that follows the syrup and ABA supplementation (red dotted line) from the feeder until reaching the brood. $\mathbf{b}$ ABA content in L5 larvae and newly emerged workers $\left(W_{S}\right)$. The measurements were repeated for three times with ten individuals in each measurement. $D W$ dry weight.

the granulocytes and plasmatocytes of ABAsupplemented larvae (Figure 3b, d). As described (Negri et al. 2014a), the implant insult induces an increase of spreading granulocytes after glass challenging in A. mellifera L5 larvae. Figure 3b shows that the implant enhances the spreading response to glass surface in granulocytes in the control group, and it was significantly increased by ABA supplementation. Granulocytes were proposed to be the first type of haemocyte to attach to a foreign object during the encapsulation response (Pech and Strand 1996; Negri et al. 2013, 2014a, b). Figure 3d shows that the number of spreading plasmatocytes was not significantly different after implantation in the control group but was significantly higher in ABAsupplemented larvae in response to the insult. The number of total haemocytes does not differ in L5 larvae from both control and ABAsupplemented groups $\left(1.8 \pm 0.5 \times 10^{6}\right.$ haemocytes $\mathrm{mL}^{-1}$ ). Figure $3 \mathrm{f}$ shows that a 2 fold increase of cell density in the agglomeration zone (see Sect. 2) of plasmatocytes from L5 larvae fed ABA-supplemented syrup compared with the control group occurs upon challenge with a glass surface.
To assess the wound-healing response in A. mellifera after the parasitism of $V$. destructor (see Sect. 2), L5 larvae were subjected to infestation with $V$. destructor in vitro, and $24 \mathrm{~h}$ later, the mites were removed and an artificial wound was performed (Figure 3g). Figure $3 \mathrm{~h}$ shows that the wound closure response in the ABAsupplemented larvae was capable of stopping the bleeding. In contrast, in all the larvae analyzed from the control group, wounds were still open and bleeding $24 \mathrm{~h}$ post-injury (Figure $3 \mathrm{~h}$ ). Figure $3 \mathrm{i}$ shows that larvae from the ABAsupplemented group present a healthy phenotype, while the control exhibited the typical aspect of sick larvae (Crailsheim et al. 2013), with browning, loss of brilliance and turgor of the skin.

\subsection{ABA enhances the immune response of worker bees}

The influence of ABA supplementation on cellular immune aptitude was also determined in $\mathrm{W}$ bees. The number of total haemocytes did not differ in newly emerged Ws from both control and ABAsupplemented groups $\left(7.49 \pm 0.91 \times 10^{6}\right.$ and 7.51 $\pm 0.89 \times 10^{6}$ haemocytes $\mathrm{mL}^{-1}$, respectively, $t$ test, 

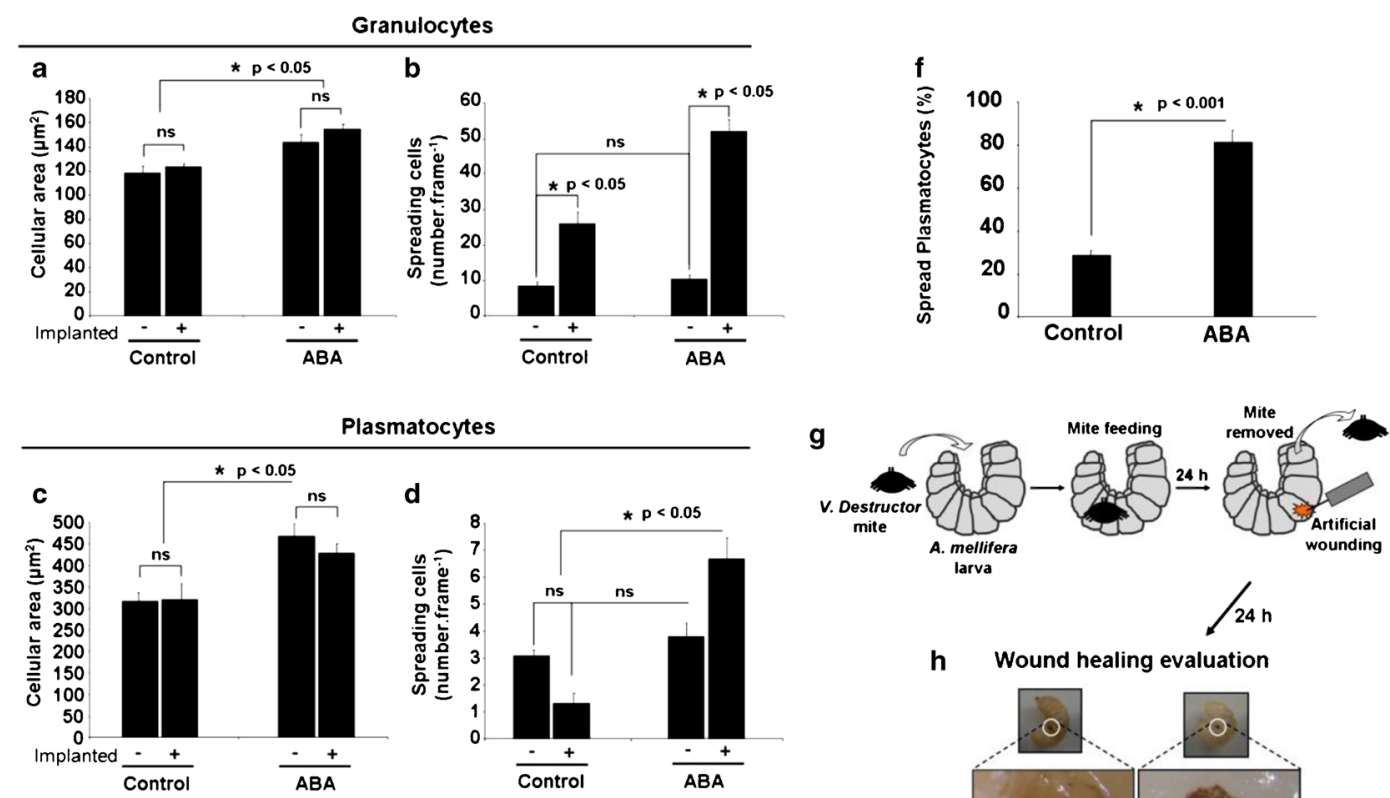

h

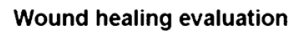

e
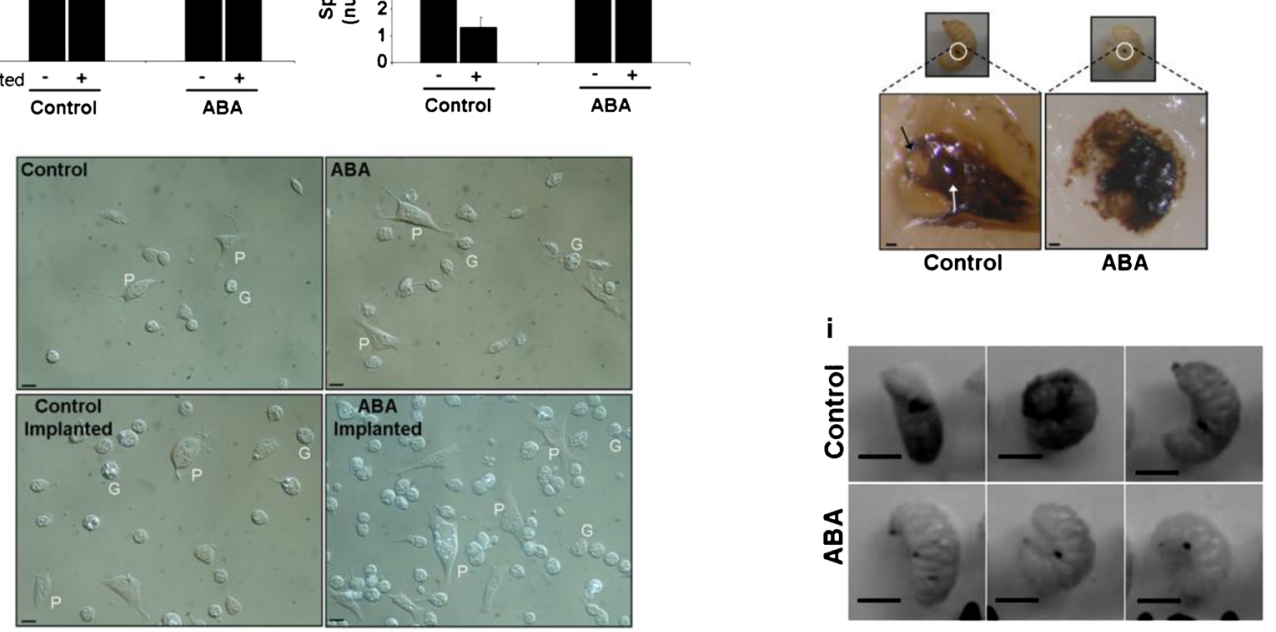

Figure 3. Effect of ABA on the immune response of L5 larvae of A. mellifera. Cellular area and number of spreading granulocytes (a, b ) and plasmatocytes (c, d ) of larvae of the control and ABA-supplemented group $24 \mathrm{~h}$ after being challenged (plus sign) or not (minus sign) with nylon implants. Asterisks mean significant differences (one-way ANOVA, $P=0.05$ ). $n s$ not significant differences between treatments. e Representative pictures of $\mathbf{a}-\mathbf{d}$. Scale bars $=10 \mu \mathrm{m}$. $G$ granulocytes, $P$ plasmatocytes. f Spread of plasmatocytes after 48 h of culture. Asterisk means significant difference (Mann-Whitney test, $P<0.001$ ). g Scheme representing the wound-healing experiment. h Images of the wounds in the L5 larvae. Black arrow: bleeding wound. White arrow: haemolymph. Scale bar $=0.1 \mathrm{~mm}$. i Images of L5 larvae after exposure to the mite $V$. destructor and the wound damage. Scale bars $=5 \mathrm{~mm}$.

$P>0.5)$. However, granulocytes from $\mathrm{Ws}$ that were reared with $\mathrm{ABA}$-supplemented syrup within the hive showed $100 \%$ more spreading than the control after $6 \mathrm{~h}$ of challenging with a glass surface (Figure 4a, b). Granulocytes from Ws from the control group reached similar values than in
ABA-supplemented Ws after $24 \mathrm{~h}$ of glass challenge, suggesting a faster immune response in granulocytes from ABA-supplemented Ws. Figure 4c shows that the supplementation with ABA enhanced the PO activity in Ws compared with the control bees, confirming that $\mathrm{ABA}$ 

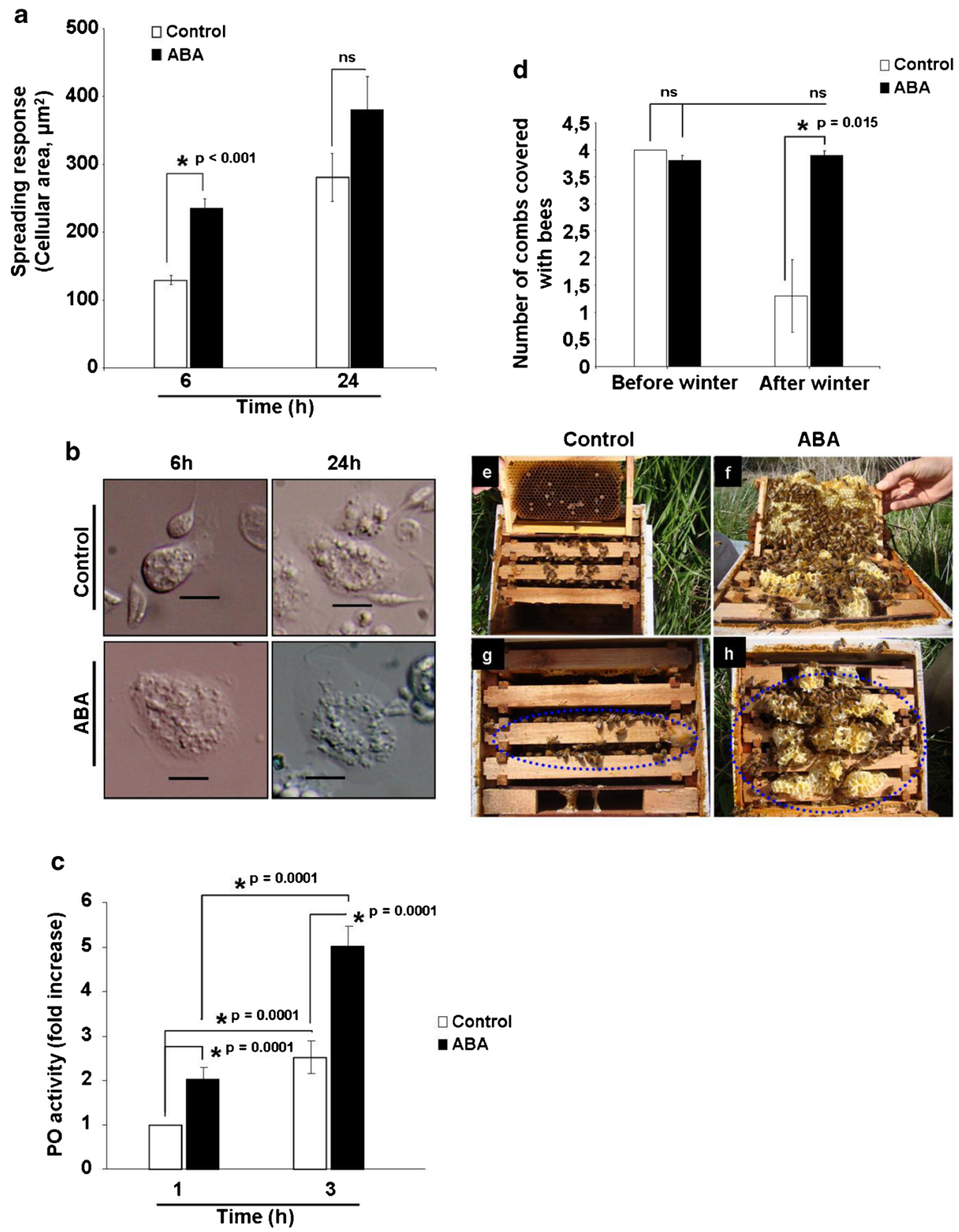

Figure 4. Effect of ABA on the immune response of $A$. mellifera worker bees and the fitness of the colonies. a Haemocyte spreading. b Representative images of granulocytes from a. Asterisk means significant difference between treatments (Mann-Whitney test, $P<0.001)$. $n s$ not significant. Scale bars $=10 \mu \mathrm{m}$. c Phenoloxidase $(P O)$ activity in extracts of Ws. The asterisk indicates significant differences (ANOVA factorial $\mathrm{a} \times \mathrm{b}, P<0.0001$ ). d Number of combs covered with adult bees in each minihive. The asterisk indicates significant difference $(P<0.015)$. $\mathbf{e}-\mathbf{h}$ Representative pictures of minihives after the winter. Blue dotted circles indicate adult bee population. 
enhances both cellular and humoral immune aptitude in Ws.

\subsection{ABA supplementation sustains the bee population in hives after the winter and increases the tolerance of honey bees to pesticides}

The influence of ABA supplementation on honey bee fitness and tolerance to low temperatures in the winter (see Supplemental Figure 2) was assessed by counting the number of combs covered with bees before and after the winter during 2012 and 2013 (Figure 4d-h). Three colonies per group were studied during 2012, and another three colonies per group were evaluated in 2013. Figure $4 \mathrm{~d}$ shows that ABAsupplemented colonies retained their initial adult population after the winter, whereas the control group diminished its population by $70 \%$. Figure $4 \mathrm{e}$ shows a control comb after the winter. An example of an ABA-supplemented comb after the same season is shown in Figure 4f. Figure 4g shows a minihive of the control group after the winter, while Figure $4 \mathrm{~h}$ shows an ABAsupplemented colony after the cold season. Additionally, supplemental Table I shows that ABA supplementation resulted in $\mathrm{W}$ individuals containing $43 \%$ more protein than the control ones.

Toxicity assays were performed confronting adult honey bees to carvacrol and OA. Figure $5 \mathrm{a}, \mathrm{b}$ shows that bees supplemented with ABA increases by $50 \%$ the $\mathrm{LC}_{50}$ for carvacrol and OA when compared with control. The positive effect of ABA on the tolerance of bees to OA was even higher in nurses than in Ws, attaining more than ten times of LC50 at $72 \mathrm{~h}$ of treatment (Figure 5b). In addition, we found that $1 \mathrm{mM}$ ABA is not toxic for bees, suggesting that they can tolerate high ABA concentrations (data not shown). In agreement, ABA has also been reported as non-toxic to bees by pesticide and veterinary experts in the Australian government (APVMA 2010).

The honey bee's catalase activity was also assayed as a measure of the ABA influence in the antioxidant capacity of bees. Supplemental
Figure 3 shows that ABA supplementation increased by $40 \%$ the catalase activity of Ws.

\section{DISCUSSION}

Given the decline of wild non-honey-bee pollinators (Whitehorn et al. 2012), the correct management of honey bees by beekeepers becomes increasingly important for the pollination service (Garibaldi et al. 2013). In this scenario, factors impacting bee health are considered worldwide concerns and should be rapidly tackled by researchers (Garibaldi et al. 2013; Dietemann et al. 2012). Here, our results highlight the close relationship between the quality of food and the health in honey bees. We presented studies directed to understand the effects of ABA on the bee's biology. The results have a significant impact since they were obtained using a combination of experiments with colonies in field under natural conditions with laboratory assays during 2 years (2012 and 2013) and using an ABA concentration in the range found in honey (Lipp 1990; Tuberoso et al. 2010). Our findings indicated that supplementing syrup with ABA resulted in a successfully dietary supplement for the colony. Indeed, we can say that the ABA supplementation worked systemically because ABA was detected in L5 larvae and adults in that their guts had been extracted before measuring ABA content. The dose of ABA $(10 \mu \mathrm{M}$; $\left.2.66 \mu \mathrm{g} \mathrm{g}^{-1}\right)$ was chosen following the analysis made by Lipp (1990) on the concentration of tt$\mathrm{ABA}+\mathrm{ct}-\mathrm{ABA}$ reported in different honey from different origins (between 0.1 and $7 \mu \mathrm{g} \mathrm{g}^{-1}$ of fresh weight, FW). The ABA concentration that was used for supplementation of minihives was also within the range of the one reported in nectar harvested by bees and in honey derived from specific flowers (Tuberoso et al. 2010). In addition, it was much less than the high $360 \mu \mathrm{g} \mathrm{g}^{-1}$ (FW) concentration of ABA found in honey from the strawberry tree (Arbutus unedo L.). Interestingly, this honey has been employed for curative purposes in ancient Greek and Roman cultures (Tuberoso et al. 2010). A relevant observation is that newly emerged Ws contained almost 7-fold more ABA than L5 larvae (Figure 2b) in both control and ABA-supplemented groups. This was an important finding since during the 12-day 


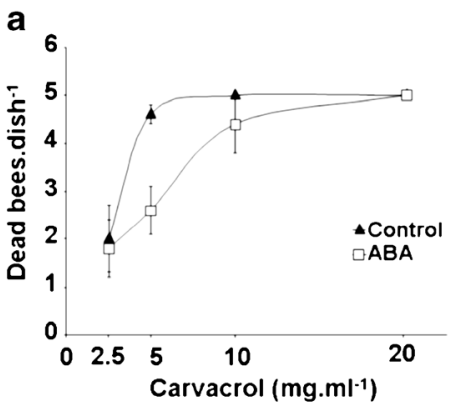

\begin{tabular}{lcc}
\hline \multicolumn{3}{l}{ Lethal concentration $50\left(\mathrm{mg} \cdot \mathrm{ml}^{-1}\right)$ for Carvacrol at $24 \mathrm{~h}$} \\
\hline & Control $\triangle$ & ABA $\square$ \\
\hline LC50 $\left(\mathrm{mg} \cdot \mathrm{ml}^{-1}\right)$ & $2.92(2.22-3.52)$ & $4.39(2.94-5.76)$ \\
\hline
\end{tabular}

\section{b}

\begin{tabular}{lcccccc}
\hline \multicolumn{6}{c}{ Lethal concentration $50(\%$ WN $)$ for Oxalic Acid } \\
\hline Time $(\mathrm{h})$ & \multicolumn{2}{c}{24} & \multicolumn{2}{c}{48} & \multicolumn{2}{c}{72} \\
\hline & NEW & Nurse & NEW & Nurse & NEW & Nurse \\
\hline Control & 2.61 & 1.29 & 2.11 & 0.9 & 1.87 & 0.4 \\
& $(1.95-3.25)$ & $(0.63-1.87)$ & $(1.61-2.58)$ & $(0.33-1.37)$ & $(1.01-2.5)$ & $(0.01-0.94)$ \\
\hline ABA & 5.16 & 5.9 & 4.54 & 5.1 & 4.41 & 4.52 \\
& $(1.81-8.85)$ & $(1.04-5.08)$ & $(2.62-5.8)$ & $(2.81-5.6)$ & $(2.18-5.37)$ & $(3.3-5.23)$ \\
\hline
\end{tabular}

Figure 5. Effect of ABA on the tolerance of the bees to carvacrol and oxalic acid. a Bioassay I: total exposure to carvacrol $\left(2.5,5,10\right.$ and $\left.20 \mathrm{mg} \mathrm{mL}^{-1}\right)$. Lethal concentration 50 (LC50) after $24 \mathrm{~h}$. Between parentheses: $95 \%$ of confidence fiducially limits. b Bioassay II: oxalic acid (OA) at concentrations of $0,1,3,5$ and $7 \%(w / v)$ added to syrup alone and syrup containing $50 \mu \mathrm{M}$ ABA. LC50 after 24, 48 and $72 \mathrm{~h}$. Between parentheses: $95 \%$ of confidence fiducially limits.

transition between L5 larvae and Ws, bees did not receive any food; however; Ws increased 7-fold their ABA. This strongly suggests an endogenous capacity of bees to generate ABA. In supporting this suggestion, honey bees possess the genetic information required for the indirect ABA biosynthesis that is found in plants, like the enzyme carotenoid isomeroxygenase 1 (CCD1; XP_394000.3; Honey Bee Genome Sequencing Consortium 2006) sharing $36 \%$ identity and $56 \%$ similarity with the Arabidopsis counterpart 9-cis epoxy-carotenoid dioxygenase (NCED) (Tossi et al. 2012). In addition, the enzymes CCDs that also participate in retinoic acid biosynthesis are present and are functional in honey bees (Evans and Wheeler 1999; Barchuk et al. 2007). Here, we show that the effects of ABA appear to be pleiotropic not only on individual bees but also on entire colonies. In other animal systems, the mechanism behind the ABA biological effect involves $A N$ increase of cytosolic $\mathrm{Ca}^{2+}$ concentration and the presence of a membranebound receptor named lanthionine synthetase Clike 2 (LANCL-2). BLAST searches against expressed tag database from GenBank were used to know the putative expression of LANCL-2 in A. mellifera. A homolog gene of LANCL-2 is present in the bee genome (Honey Bee Genome Sequencing Consortium 2006). There exist seven transcriptional variants of LANCL-2 reported in the EST database of honey bee, and variants $1,3,5$ and 6 share between 33 and $46 \%$ sequence identity and 53 to $71 \%$ similarity with the human counterpart (BLASTp). These data strongly suggest the presence of an expressed putative receptor for $\mathrm{ABA}$ and an operative mechanism underlying the ABA biological activity in A. mellifera.

One of the main threats affecting $A$. mellifera colonies worldwide is the ectoparasitic mite $V$. destructor. This parasite feeds from A. mellifera larvae haemolymph diminishing its 
fitness due to blood extraction and virus vectorization, such as the deformed wing virus (DWV) (Martin et al. 2012; Gätschenberger et al. 2013). To achieve long periods of haemolymph sucking, $V$. destructor secretes anticoagulant factors in its saliva (Richards et al. 2011). This anticoagulant effect is essential to the mite reproductive phase since $V$. destructor broods are incapable of puncturing the larvae cuticle and need their mother to pierce it (Rosenkranz et al. 2010). Thus, the wounds caused by $V$. destructor in A. mellifera larvae represent a crucial threat to be faced by the bee's immune response. Recently, we have reported that haemocytes of $A$. mellifera respond to foreign surfaces in vitro and play a role in the woundhealing response after artificial injuries generated with nylon implants (Negri et al. 2013, 2014a, b). Nylon implants in insects are also considered an immune challenge that triggers non-self recognition, followed by encapsulation and wound healing (Wilson-Rich et al. 2008; Negri et al. 2014a). In addition, the agglomeration of haemocytes is essential for the wound-healing process in insects (Richards et al. 2011). Since an augmented agglomeration response was observed in haemocytes from ABA-supplemented larvae, we tested the wound closure response in ABA-supplemented larvae after $V$. destructor parasitism. As a consequence, the anticoagulant effect of the mite saliva interfered the healing response. Interestingly, after $24 \mathrm{~h}$ that an artificial injury was performed with a needle, the ABAsupplemented larvae were able to stop bleeding, while control larvae were still bleeding. The goal in these experiments was to study the effect of ABA on the wound-healing response under unfavourable conditions.

The phenoloxidase (PO) activity is important in the melanization response during wound healing in insects (Gillespie and Kanost 2006). Since PO activity was reported to be stronger in adult bees compared to larvae, it was measured in Ws (Wilson-Rich et al. 2008). Results showed that ABA supplementation increased PO activity in honey bees.

Organic pesticides as well as synthetic drugs have been reported causing long-term impact on honey bee health (Higes et al. 1999; Mao et al.
2013). Cellular immune responses are believed to play an important role in defending insects against pesticide poisonings (James and Xub 2012). Since augmented cellular immunocompetence was observed in ABA-supplemented bees, toxicity assays were performed to evaluate the aptitude of bees to confront pesticides. Carvacrol is a monoterpenoid phenol that inhibits the growth of several bacteria, e.g., Escherichia coli and Bacillus cereus (Du et al. 2008). OA is an organic acid conventionally used in apiculture to control $V$. destructor, even though it has been demonstrated to have adverse effects on the survival of honey bee colonies (Hatjina and Haristos 2005). The toxicity assays indicated that, in comparison with control bees, ABA supplementation allowed honey bees to tolerate almost twice the concentration of carvacrol and OA. $p$-Coumaric acid, a natural compound present in pollen and in honey, has been recently reported to induce the expression of detoxification genes related to bee's tolerance to pesticides (Mao et al. 2013). ROS are involved in the toxicity of various pesticides (Bagchi et al. 1995). OA and carvacrol induce the generation of ROS in cells (Kim et al. 2008; Huang et al. 2010). CAT is a very important enzyme in protecting cells from oxidative damage by ROS (Chelikani et al. 2004), and here, we show that ABA supplementation increased CAT activity in bees. Thus, the increased tolerance to carvacrol and OA related with ABA supplementation could be at least partially explained by an augmented antioxidant aptitude.

The mechanism underlying the ABAdependent increase of immunity and tolerance to pesticides is not fully understood yet. As described above, ABA increased the activities of PO and catalase by 2.5 and 1.5 -fold, respectively, without any challenges. If it is assumed that this increase represents a metabolic cost for the bees, it will be interesting to know how bees attain a balance between costs and benefits provided by ABA in a long-term analysis, at both individual and colony levels.

In order to complement the results showed in this work, several questions linked to nutritional status of bees should be addressed in the future: (i) Is the naturally occurring ABA in honey used deliberately by bees in their diet to enhance the 
colony health and to protect themselves and (ii) if beekeepers take honey from the hives containing ABA and replace partially it with syrup containing no ABA, will the bees be impacted in health and behaviour by the diminution in ABA concentration in bee's diet? It is possible that since the beginning of the co-evolution between plants and bees, the plant hormone ABA allows $A$. mellifera to confront stressors. We would like emphasize here the importance of plants in supplying honey bees not only with sugars in nectar as an energy source but also with other biologically active molecules like ABA. In this sense, it has been found that resins collected by bees affect the expression of immunity genes (Simone et al. 2009) and also that bees self-medicate by increasing resin collection in response to fungal infections (Simone-Finstrom and Spivak 2012). In another approach, Niu et al. (2011) demonstrated that propolis extracts reduce toxicity of mycotoxins. All these pieces of work demonstrated that it is imperative to find alternative ways of pesticide usage and attenuate the emergence of resistant plagues (Maggi et al. 2010b). This work offers a new tool for beekeepers and researchers for developing an innovative and environmentally friendly management of honey bee colonies. We anticipate our assays to be of relevance for studies associating $A$. mellifera 's immune aptitude with the quality and composition of their diet.

\section{ACKNOWLEDGMENTS}

The authors want to thank Dr Mary M. Barile from Columbia University at Missouri for having kindly corrected the text of the manuscript. This research was supported by grants PICT-2011 No. 2383 to LL from ANPCyT, PIP-2011 to LL from Consejo Nacional de Investigaciones Científicas y Técnicas (CONICET) and PIP-2011 No. 0162 to MM from CONICET. The authors would like to thank CONICET and the UNMdP for financial support. PN is a doctoral fellow from CONICET, Argentina.

$L$ 'acide abscissique amplifie la réponse immunitaire chez Apis mellifera et contribue à la bonne santé de la colonie
ABA / Apidae / défense immunitaire / régime alimentaire

\section{Abscisinsäure verstärkt die Immunantwort in Apis mellifera und trägt zur Fitness der Kolonie bei}

Apidae / Abscisinsäure / Immunanlage

\section{REFERENCES}

Adie, B.A.T., Pérez-Pérez, J., Pérez-Pérez, M.M., Godoy, M., Sánchez-Serrano, J., Schmelz, E.A., Solanoa, R. (2007) ABA is an essential signal for plant resistance to pathogens affecting JA biosynthesis and the activation of defences in Arabidopsis. The Plant Cell 19, 1665-1681

Alaux, C., Ducloz, F., Crauser, D., Le Conte, Y. (2010) Diet effects on honeybee immunocompetence. Biol. Lett. . doi:10.1098/rsbl.2009.0986

Aliano, N.P., Ellis, M.D., Siegfried, B.D. (2006) Acute contact toxicity of oxalic acid to Varroa destructor (Acari: Varroidae) and their Apis mellifera (Hymenoptera: Apidae) hosts in laboratory bioassays. J. Econ. Entomol. 99, 1579-1582

Anderson, D., Trueman, J. (2000) Varroa jacobsoni (Acari: Varroidae) is more than one species. Exp. Appl. Acarol. 24, 165-189

Bagchi, D., Bagchi, M., Hassoun, E.A., Stohs, S.J. (1995) In vitro and in vivo generation of reactive oxygen species, DNA damage and lactate dehydrogenase leakage by selected pesticides. Toxicology 104, 129-140

Barchuk, A., Cristino, A., Kucharski, R., Costa, L., Simões, Z., Maleszka, R. (2007) Molecular determinants of caste differentiation in the highly eusocial honeybee Apis mellifera. BMC Dev. Biol. 7, 70. doi:10.1186/ 1471-213X-7-70

Brodschneider, R., Crailsheim, K. (2010) Nutrition and health in honey bees. Apidologie 41, 278-294

Bruzzone, S., Moreschi, I., Usai, C., Guida, L., Damonte, G., Salis, A., Scarfî, S., Millo, E., De Flora, A., Zocchi, E. (2007) Abscisic acid is an endogenous cytokine in human granulocytes with cyclic ADP-ribose as second messenger. Proc. Nat. Acad. Sci 104, 5759-5764

Bruzzone, S., Basile, G., Mannino, E., Sturla, L., Magnone, M., et al. (2012) Autocrine abscisic acid mediates the UV-B-induced inflammatory response in human granulocytes and keratinocytes. J Cell Physiol 227, 2502-2510

Bryden, J., Gill, R.J., Mitton, R.A.A., Raine, N.E., Jansen, V.A.A. (2013) Chronic sublethal stress causes bee colony failure. Ecol. Lett. 16(12), 1463-1469

Chelikani, P., Fita, I., Loewen, P.C. (2004) Diversity of structures and properties among catalases. Cell. Mol. Life Sci. 61 (2), 192-208 
Crailsheim K., Brodschneider R., Aupinel P., Behrens D., Genersch E., Vollmann J., Riessberger-Gallé U. (2013) Standard methods for artificial rearing of Apis mellifera larvae. In V Dietemann; J D Ellis; P Neumann (Eds) The COLOSS BEEBOOK, Volume I: standard methods for Apis mellifera research. J. Apic. Res., 52(1), 1-15.

Davies, D.H., Preston, T.M. (1985) The behaviour of insect plasmatocytes in vitro: changes in morphology during settling, spreading, and locomotion. J. Exp. Zool. 236, 71-82

Dean, P., Richards, E.H., Edwards, J.P., Reynolds, S.E., Charnley, K. (2004) Microbial infection causes the appearance of hemocytes with extreme spreading ability in monolayers of the tobacco hornworm Manduca sexta. Dev. Comp. Immunol. 28, 689-700

Decourtye, A., Mader, E., Desneux, N. (2010) Landscape enhancement of floral resources for honey bees in agro-ecosystems. Apidologie 41, 264-277

Dietemann, V., Pflugfelder, J., Anderson, D., Charrière, J.D., Chejanovsky, N., et al. (2012) Varroa destructor: research avenues towards sustainable control. J. Apic. Res. 51, 125-132

Du, W.X., Olsen, C.W., Avena-Bustillos, R.J., McHugh, T.H., Levin, C.E., Friedman, M. (2008) Storage stability and antibacterial activity against Escherichia coli O157:H7 of carvacrol in edible apple films made by two different casting methods. J. Agric. Food Chem. 56, 3082-3088

Durgbanshi, A., Arbona, V., Pozo, O., Miersch, O., Sancho, J.V., Gómez-Cadenas, A. (2005) Simultaneous determination of multiple phytohormones in plant extracts by liquid chromatography-electrospray tandem mass spectrometry. J. Agric. Food Chem. 53 , 8437-8442

Eleftherianos, I., Xu, M., Yadi, H., Ffrench-Constant, R.H., Reynolds, S.E. (2009) Plasmatocyte-spreading peptide (PSP) plays a central role in insect cellular immune defences against bacterial infection. J. Exp. Biol. 212, 1840-1848

Evans, J., Wheeler, D. (1999) Differential gene expression between developing queens and workers in the honey bee, Apis mellifera. PNAS 96, 5575-5580

Ferreres, F., Andrade, P., Tomás-Barberán, F. (1996) Natural occurrence of abscisic acid in heather honey and floral nectar. J. Agric. Food Chem. 44, 2053-2056

Garibaldi, L.A., Steffan-Dewenter, I., Winfree, R., Aizen, M.A., Riccardo, B.R., et al. (2013) Wild pollinators enhance fruit set of crops regardless of honey bee abundance. Science 339, 1608-1611

Gätschenberger, H., Azzami, K., Tautz, J., Beier, H. (2013) Antibacterial immune competence of honey bees (Apis mellifera $)$ is adapted to different life stages and environmental risks. Plos ONE 8 (6), e66415. doi:10.1371/ journal.pone.0066415

Gillespie, J.P., Kanost, M.R. (2006) Biological mediators of insect immunity. Annu. Rev. Entomol. 42, 611-643

Harbo, J.R. (1985) Effect of population size on brood production, worker survival and honey gain in colonies of honey bees. J. Apic. Res. 25, 22-29
Hatjina, F., Haristos, L. (2005) Indirect effects of oxalic acid administered by trickling method on honey bee brood. J. Apic. Res. 44, 172-174

Higes, M., Meana, A., Suárez, M., Llorente, J. (1999) Negative long-term effects on bee colonies treated with oxalic acid against Varroa jacobsoni . Apidologie $\mathbf{3 0}$, 289-292

Honey bee genome Sequencing Consortium (2006) Sequencing honey bee genome. Insights into social insects from the genome of the honey bee Apis mellifera. Nature 443, 931-949

Huang, T.C., Lin, T.Y., Chuang, K.P. (2010) Carvacrol has the priming effects of reactive oxygen species (ROS) production in C6 glioma cells. Food Agric. Immunol. 21, 47-55

James, R.R., Xub, J. (2012) Mechanisms by which pesticides affect insect immunity. J. Invertebr. Pathol. 109, 175-182

Keasar, T., Shihadeh, S., Shmida, A., Majali, N., Weil, D., Reuven, N. (2007) An evaluation of mini-nucleus honey bee hives for pollination of honeydew melons in enclosures. J. Apic. Res. 46, 264-268

Kim, K.S., Min, J.Y., Dickman, M.B. (2008) Oxalic acid is an elicitor of plant programmed cell death during Sclerotinia sclerotiorum disease development. Mol. Plant Microbe Interact. 21, 605-612

Kukielka D., Esperón F., Higes M., Sánchez-Vizcaíno J.M. (2008) A sensitive one-step real-time RT-PCR method for detection of deformed wing virus and black queen cell virus in honeybee Apis mellifera. J. Virol. Methods. 147, 275-281

Lindberg, C., Melathopoulus, A., Winston, M. (2000) Laboratory evaluation of miticides to control Varroa jacobsoni, a honey bee parasite. J. Econ. Ent. 93, 189-198

Lipp, J. (1990) Nachweis und herkunft von abscisinsäure und prolin in honig. Apidologie 21, 249-259

Maggi, M., Damiani, N., Ruffinengo, S., De Jong, D., Principal, J., Eguaras, M. (2010a) Brood cell size of Apis mellifera modifies the reproductive behaviour of Varroa destructor. Exp. Appl. Acarol. 50, 269-79

Maggi, M., Ruffinengo, S., Negri, P., Eguaras, M. (2010b) Resistance phenomena to amitraz from populations of the ectoparasitic mite Varroa destructor of Argentina. Parasitol. Res. 107, 1189-1192

Maggi, M.D., Ruffnengo, S.R., Gende, L.B., Sarlo, E.G., Eguaras, M.J., Bailac, P.N., Ponzi, M.I. (2011) Laboratory evaluations of Syzygium aromaticum (L.) Merr. et Perry essential oil against Varroa destructor. J Essential Oil Res 22, 119-122

Maggi, M., Negri, P., Plischuk, S., Szawarski, N., De Piano, F., De Feudis, L., Eguaras, M., Audisio, C. (2013) Effects of the organic acids produced by a lactic acid bacterium in Apis mellifera colony development, Nosema ceranae control and fumagillin efficiency. Vet. Microbiol . doi:10.1016/j.vetmic.2013.07.030

Mao, W., Schuler, M.A., Berenbaum, M.R. (2013) Honey constituents up-regulate detoxification and immunity 
genes in the western honey bee Apis mellifera. Proc. Nat. Acad. Sci. . doi:10.1073/pnas.1303884110

Martin, S., Highfield, A., Brettell, L., Villalobos, E., Budge, G., Powell, M., Nikaido, S., Schroeder, D. (2012) Global Honey Bee Viral Landscape Altered by a Parasitic Mite. Science 336, 1304-1306

Nardi, J.B., Pilas, B., Bee, C.M., Zhuang, S., Garsha, K., Kanost, M.R. (2006) Neuroglian-positive plasmatocytes of Manduca sexta and the initiation of hemocyte attachment to foreign surfaces. Dev. Comp. Immunol. 30 , 447-462

Negri, P., Maggi, M., Correa-Aragunde, N., Brasesco, C., Eguaras, M., Lamattina, L. (2013) Nitric oxide participates at the first steps of Apis mellifera cellular immune activation in response to non-self recognition. Apidologie 44, 575-585

Negri, P., Quintana, S., Maggi, M., Szawarski, N., Lamattina, L., Eguaras, M. (2014a) Apis mellifera hemocytes generate increased amounts of nitric oxide in response to wounding/encapsulation. Apidologie . doi:10.1007/s13592-014-0279-0

Negri P., Maggi M. Szawarski N., Lamattina L., Eguaras M. (2014b) Apis mellifera hemocytes in-vitro: What type of cells are they? Functional analysis before and after the pupae metamorphosis black box. J. Apic. Res., In Press .

Niu, G., Johnson, R., Berenbaum, M. (2011) Toxicity of mycotoxins to honeybees and its amelioration by propolis. Apidologie 42, 79-87

Pech, L., Strand, M. (1996) Granular cells are required for encapsulation of foreign targets by insect haemocytes. J. Cell Sci. 109, 2053-2060

Porrini, M.P., Audisio, M.C., Sabaté, D.C., Ibarguren, C., Medici, S.K., Sarlo, E.G., Garrido, P.M., Eguaras, M.J. (2011) Effect of bacterial metabolites on microsporidian Nosema ceranae and on its host Apis mellifera. Parasitol. Res. 107, 381-388

Potts, S.G., Biesmeijer, J.C., Kremen, C., Neumann, P., Schweiger, O., Kunin, W.E. (2010) Global pollinator declines: trends, impacts and drivers. Trends Ecol. Evol. 25, 345-353

Richards, E.H., Jones, B., Bowman, A. (2011) Salivary secretions from the honey bee mite, Varroa destructor: effects on insect haemocytes and preliminary biochemical characterization. Parasitology 138, 602-608

Rosenkranz, P., Aumeier, P., Ziegelmann, B. (2010) Biology and control of Varroa destructor. J. Invertebr. Pathol. 103, 96-119

Schaefer, H.M., Schaefer, V., Levey, D.J. (2004) How plant-animal interactions signal new insights in communication. Trends Ecol. Evol. 19, 577-584

Simone, M., Evans, J., Spivak, M. (2009) Resin collection and social immunity in honey bees. Evolution 63 , 3016-3022

Simone-Finstrom, M., Spivak, M. (2012) Increased Resin Collection after Parasite Challenge: A Case of SelfMedication in Honey Bees? PLoS ONE 7 (3), e34601. doi:10.1371/journal.pone.0034601

Strand, M.R. (2008) The insect cellular immune response. Insect Sci. 15, 1-14

Tossi, V., Cassia, R., Bruzzone, S., Zochi, E., Lamattina, L. (2012) ABA Says NO to UV-B: A Universal Response? Trends Plant Sci. 17, 510-517

Tuberoso, C.I., Bifulco, E., Caboni, P., Cottiglia, F., Cabras, P., Floris, I. (2010) Floral markers of strawberry tree (Arbutus unedo L.) honey. J. Agric. Food Chem 58, 384-389

APVMA (Australian Pesticides and Veterinarian Medicines Authority), on the evaluation of the new active sabscisic acid in the product ProTone SG Plant Growth Regulator Soluble Granule, product number 63314, August 2010.

Whitehorn, P.R., O'Connor, S., Wackers, F.L., Goulson, D. (2012) Neonicotinoid pesticide reduces bumble bee colony growth and queen production. Science 336, 351-352

Wilson-Rich, N., Dres, D., Starks, P. (2008) The ontogeny of immunity: Development of innate immune strength in the honey bee (Apis mellifera ). J. Insect Physiol. 54, 1392-1399

Yang X., Cox-Foster D.L. (2005) Impact of an ectoparasite on the immunity and pathology of an invertebrate: evidence for host immunosuppression and viral amplification. Proc. Natl. Acad. Sci. USA. 102, 7470-7475 